\title{
Brucellosis caused by the wood rat pathogen Brucella neotomae: two case reports
}

\author{
Juan M. Villalobos-Vindas ${ }^{1}$, Ernesto Amuy ${ }^{1}$, Elías Barquero-Calvo ${ }^{2}$, Norman Rojas ${ }^{3}$, Carlos Chacón-Díaz ${ }^{3}$, \\ Esteban Chaves-Olarte ${ }^{3}$, Caterina Guzman-Verri ${ }^{2}$ and Edgardo Moreno ${ }^{2,4^{*}}$
}

\begin{abstract}
Background: Brucellosis is a chronic bacterial disease caused by members of the genus Brucella. Among the classical species stands Brucella neotomae, until now, a pathogen limited to wood rats. However, we have identified two brucellosis human cases caused by B. neotomae, demonstrating that this species has zoonotic potential.

Cases presentation: Within almost 4 years of each other, a 64-year-old Costa Rican white Hispanic man and a 51-year-old Costa Rican white Hispanic man required medical care at public hospitals of Costa Rica. Their hematological and biochemical parameters were within normal limits. No adenopathies or visceral abnormalities were found. Both patients showed intermittent fever, disorientation, and general malaise and a positive Rose Bengal test compatible with Brucella infection. Blood and cerebrospinal fluid cultures rendered Gram-negative coccobacilli identified by genomic analysis as B. neotomae. After antibiotic treatment, the patients recovered with normal mental activities.

Conclusions: This is the first report describing in detail the clinical disease caused by B. neotomae in two unrelated patients. In spite of previous claims, this bacterium keeps zoonotic potential. Proposals to generate vaccines by using $B$. neotomae as an immunogen must be reexamined and countries housing the natural reservoir must consider the zoonotic risk.
\end{abstract}

Keywords: Brucella neotomae, Brucellosis, Neurobrucellosis, Brucella, Zoonosis

\section{Background}

Brucellosis is an infectious bacterial disease of animals and a relevant zoonosis [1]. Four Brucella species have been reported to be the main cause of human infections: Brucella abortus, Brucella melitensis, Brucella suis, and Brucella canis [1]. In addition, rare Brucella isolates have been reported in human cases, including some strains similar to those isolated from marine mammals [2-4]. In all these cases the identification of Brucella organisms has relied on molecular methods, with no epidemiological information that could explain the source for transmission to humans.

\footnotetext{
* Correspondence: edgardo.moreno.robles@una.cr

${ }^{2}$ Programa de Investigación en Enfermedades Tropicales, Universidad Nacional, Heredia, Costa Rica

${ }^{4}$ Instituto Clodomiro Picado, Universidad de Costa Rica, San José, Costa Rica Full list of author information is available at the end of the article
}

Brucella neotomae, first isolated in 1957 in desert wood rats of the genus Neotomae in the United States of America (USA) [5], has been considered for 60 years a non-zoonotic bacterium and therefore of no risk to humans. However, B. neotomae has been isolated in the organs of wood rats and has been shown to display pathogenicity for mice [6]. Moreover, whole genome analysis of $B$. neotomae has revealed that this bacterium possesses the same virulence arsenal as the classic zoonotic brucellae [7]. Therefore, its potential as a human pathogen cannot be ruled out a priori. Here we describe two clinical cases of brucellosis caused by $B$. neotomae and discuss the medical and epidemiological implications of our findings. 


\section{Case presentation}

\section{Case 1}

A 64-year-old Costa Rican white Hispanic man, an inhabitant from the Central Valley of Costa Rica (1000 m altitude), presented to a Costa Rican Social Security Hospital. He was hypertensive. On arrival, he presented a lung infection and a stroke with an intraparenchymal hemorrhage of the right basal ganglia with extension to the ipsilateral lateral ventricle, as demonstrated by computed axial tomography. He was treated with penicillin G ( $10^{6}$ units/intramuscular/7 days) and received medical intervention for 7 days. After this period he recovered with no recurrence of his illness.

He presented at the hospital again, 13 months after this event, with left hemiparesis, headache, disorientation, dysarthria, lethargy, and intermittent fever. He did not respond to questions and did not follow instructions. He was kept at the hospital for examination, observation, and treatment. His hematological and biochemical parameters were within normal ranges, including leukocyte counts and cell morphology. A C-reactive protein test was negative. An abdominal ultrasound did not demonstrate alterations and other tests revealed normal size and function of his liver and spleen. Diagnostic findings by an echocardiogram did not reveal any vegetation or other sequelae of infectious endocarditis. Computed axial tomography showed sequelae related to the stroke he had 1 year before.

After hospitalization, he had some improvement, but the intermittent fever remained (Fig. 1). After 12 days, a blood culture for the presence of bacteria was performed in blood agar, with negative results after 3 days of incubation. At the fourth day, a second blood culture was taken and cerebrospinal fluid extracted, analyzed, and cultured in blood agar. Concomitantly, he received treatment with 1 gram of cefotaxime by an intravenous route every 8 hours. The cerebrospinal fluid was cloudy with protein levels of $220 \mathrm{mg} / \mathrm{dL}, 20$ erythrocytes $/ \mathrm{mm}^{3}$, and 222 leukocytes $/ \mathrm{mm}^{3}$ with $94 \%$ lymphocytes. The first blood culture was kept up to 1 week, with negative results. He showed positive agglutination in Rose Bengal test for brucellosis. After 3 days of incubation of the second blood culture, Gram-negative coccobacilli compatible with Brucella were isolated. Likewise, Gram-negative coccobacilli similar to that from the blood culture were also isolated in blood agar from the cerebrospinal fluid, after 3 days. The isolates were highly sensitive to a panel of antibiotics used to treat brucellosis [8]. The cefotaxime treatment was interrupted and a combination of doxycycline (100 mg/12 hours by the oral route) and streptomycin $(750 \mathrm{mg} / 24$ hours, by the intramuscular route) was given for 12 and 4 weeks, respectively. After antibiotic treatment, he recovered with no fever and with normal mental activities, but remained with slight left hemiparesis sequelae.

In the course of this study, no species was assigned for the isolated Brucella species. Five years later, the bacterium was identified as $B$. neotomae by multiple-locus variable number tandem repeat analysis of 16 sequences (MLVA16) and whole genome sequencing [7].

\section{Case 2}

A 51-year-old Costa Rican white Hispanic man from Puntarenas, East Pacific coast of Costa Rica, presented to the local Social Security Hospital 3 years and 11 months after Case 1. He presented a recurrent headache, disorientation, general muscle and joint pain, weight loss, cough, anorexia, and intermittent nocturnal fever of 3 weeks of evolution. Since he had a previous clinical history of dengue fever and lived in an endemic dengue region on the Pacific coast of Costa Rica, he was hospitalized as a possible case of dengue. On general

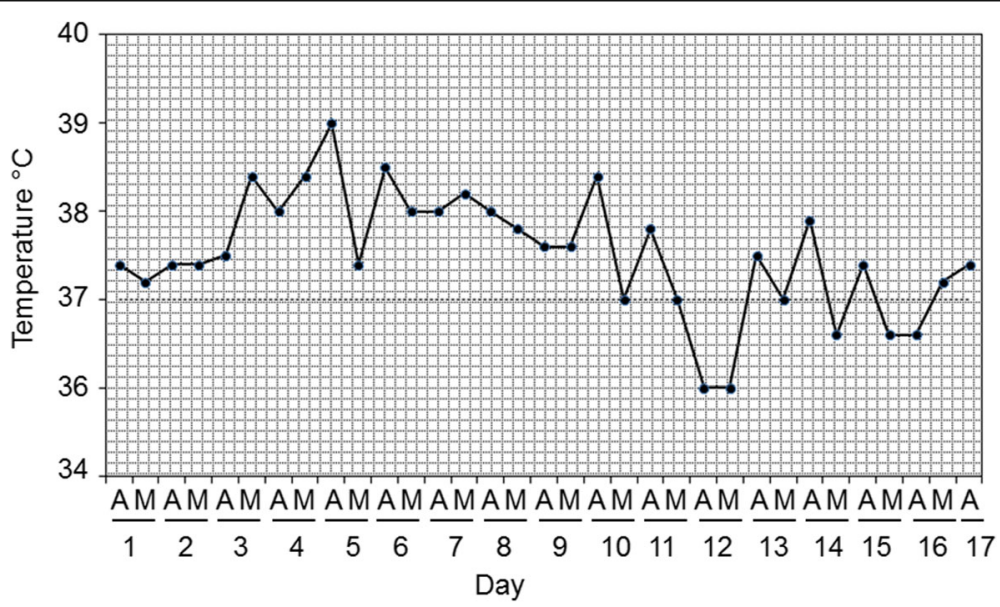

Fig. 1 Clinical chart displaying the (undulant) intermittent fever experienced during hospitalization by patient of case 1. Temperature was taken during the morning and afternoon with 12 hours difference. A afternoon, $M$ morning 
examination, he did not present rash, adenopathies, abdominal pain, or visceral enlargement. Blood tests showed normal leukocyte counts. With the exception of a mild thrombocytopenia, a hemogram was within normal parameters. A C-reactive protein test was negative. The results of a differential molecular diagnosis by polymerase chain reaction (PCR) for dengue, toxoplasmosis, cytomegalovirus, malaria, and Epstein-Barr virus were all negative. He showed positive agglutination in Rose Bengal test for brucellosis. Gram-negative coccobacilli compatible with Brucella species were recovered from blood after 3 days of bacteriological culture in blood agar. The isolate was highly sensitive to a panel of antibiotics used to treat brucellosis [8]. Following this, he was treated with doxycycline (100 mg/12 hours by the oral route) and rifampin (900 mg/day orally) for 45 days. For the next days he showed considerable health improvement and after 6 days of hospitalization he was released. A follow up in local medical clinics was established. After the end of the antibiotic treatment, the Rose Bengal test became negative and he recovered with no sequelae.

Initially, the isolated etiological agent was misidentified as $B$. abortus by bacteriological and biochemical tests. However, 2 years later, when the isolates were analyzed by MLVA16 and whole genome sequencing, it became evident that the bacterial strains belonged to $B$. neotomae species [7].

\section{Discussion}

Here, we have described in detail two human clinical cases caused by $B$. neotomae. In a previous work, we reported the phylogenetic and genomic characteristics of the $B$. neotomae strains isolated in these two human cases [7]. This is relevant, considering that the identification of B. neotomae is not straightforward and to distinguish it from other Brucella species by common bacteriological techniques is difficult [9]. Several years after the isolation of the etiological agents causing brucellosis in these two patients, the Brucella species were unambiguously identify following MLVA16 and whole genome sequence analysis [6]. Therefore, these techniques are valuable tools for the recognition of nonconventional Brucella strains.

The outcomes of the disease in the two B. neotomae clinical cases described here, do not depart from other classical brucellosis cases reported elsewhere [1, 10-12]. In fact, the course of zoonotic brucellosis, in general, is chronic with intermittent fever and a broad range of non-pathognomonic symptoms. In some instances, the bacterium is also capable of crossing the blood-brain barrier and invading the brain, causing neurobrucellosis [13], as in one of the cases presented here.

From the clinical perspective and at a glance, brucellosis is of difficult diagnosis and commonly is confused with other diseases that also cause intermittent fever. In fact, in contrast to other bacterial diseases, the course of brucellosis might not present endotoxicity signs, increase in proteins related to inflammatory processes and coagulopathies, or significant blood leukocyte changes [10-12]. However, in long-lasting cases, absolute neutropenia, thrombocytopenia, and increase of some proinflammatory proteins may be observed in approximately one third of the cases [10-12].

In tropical countries such as Costa Rica the differential diagnosis has to be carried out with dengue, chikungunya, Zika virus, malaria, and some viral infections. Moreover, classical diagnostic tests such as agglutination may be negative in a number of brucellosis cases, precluding a straightforward diagnosis [14]. For this, repeated serological testing and blood cultures and, when required, cerebrospinal fluid cultures (in the case of neurological disorders) are recommended before antibiotic treatment. Once the diagnosis has been established, treatment with a combination of two antibiotics for several weeks becomes mandatory. Fortunately, antibiotic resistance has seldom been reported in brucellosis [1] and up to now all strains, including $B$. neotomae (as demonstrated here) are susceptible to aminoglycoside and antibiotics of the tetracycline and rifampicin class.

It is intriguing how these two persons acquired $B$. neotomae infections within almost 4 years of each other and living in two different regions of the country. From the epidemiological perspective it is worth noting that in Costa Rica there are no rats of the genus Neotomae, although there are other Neotominae species such as those of the genus Reithrodontomys which are endemic [15]. Whether these rat species could serve as reservoirs for $B$. neotomae remains unknown. In any case, it is clear that human brucellosis is not limited to the socalled "classical" zoonotic Brucella species, and other members of the genus may also keep this potential. This is relevant, considering that countries such as USA and Canada (that have eradicated zoonotic Brucella species from cows, sheep, pigs, and goats) may still harbor $B$. neotomae in wood rats. Moreover, works devoted to generate vaccines by using $B$. neotomae as an immunogen $[16,17]$ must be reexamined.

\section{Conclusions}

Physicians should consider brucellosis caused by B. neotomae as one of the agents for human disease. Even in those areas where Brucella organisms have been eradicated from domestic animals, $B$. neotomae may still be a zoonotic risk. The fact that $B$. neotomae is capable of invading the brain indicates the robust pathogenic potential of $B$. neotomae. Differential diagnosis with other diseases that present with intermittent fever is necessary, whether they are in endemic or non-endemic areas. 


\section{Abbreviation}

MLVA16: Multiple-locus variable number tandem repeat analysis of 16 sequences

\section{Acknowledgements}

The authors thank the medical and laboratory personnel of Hospital Mexico and Hospital Monseñor Sanabria for their assistance in the cases. We also thank Nazareth Ruiz and Marcela Suarez for helping to obtain the clinical information.

\section{Funding}

Fondo Institucional de Desarrollo Académico (FIDA), Universidad Nacional SIA 087-17.

International Centre for Genetic Engineering and Biotechnology (ICGB) CRP-CRI16-02

\section{Availability of data and materials}

Data sharing is not applicable to this article as no data sets were generated or analyzed during the current study.

\section{Authors' contributions}

JMV-V and EA performed the clinical and laboratory studies. EB-C, NR, CC-D, EC-O, and CG-V collected the bacteriological, serological, and molecular data. EM coordinated the work and drafted the manuscript. All authors read and approved the final manuscript

\section{Ethics approval and consent to participate}

Not applicable

\section{Consent for publication}

Written informed consent was obtained from the patients for publication of this case report and any accompanying images. A copy of the written consent is available for review by the Editor-in-Chief of this journal.

\section{Competing interests}

The authors declare that they have no competing interests.

\section{Publisher's Note}

Springer Nature remains neutral with regard to jurisdictional claims in published maps and institutional affiliations.

\section{Author details}

'Caja Costarricense de Seguro Social, San José, Costa Rica. ${ }^{2}$ Programa de Investigación en Enfermedades Tropicales, Universidad Nacional, Heredia, Costa Rica. ${ }^{3}$ Centro de Investigación en Enfermedades Tropicales, Universidad de Costa Rica, Heredia, Costa Rica. ${ }^{4}$ Instituto Clodomiro Picado, Universidad de Costa Rica, San José, Costa Rica.

\section{Received: 13 June 2017 Accepted: 21 October 2017}

\section{Published online: 19 December 2017}

References

1. Moreno E. Retrospective and prospective perspectives on zoonotic brucellosis. Front Microbiol. 2014;5:213. doi:10.3389/fmicb.2014.00213.

2. Scholz HC, Nöckler $K$, Göllner C, Bahn P, Vergnaud G, Tomaso H, Al Dahouk S, Kämpfer P, Cloeckaert A, Maquart M, Zygmunt MS, Whatmore AM, Pfeffer M, Huber B, Busse HJ, De BK. Brucella inopinata sp. nov., isolated from a breast implant infection. Int J Syst Evol Microbiol. 2010;60:801-8.

3. Tiller RV, Gee JE, Lonsway DR, Gribble S, Bell SC, Jennison AV, Bates J, Coulter C, Hoffmaster AR, De BK. Identification of an unusual Brucella strain (BO2) from a lung biopsy in a 52 year-old patient with chronic destructive pneumonia. BMC Microbiol. 2010;10:23. doi:10.1186/1471-2180-10-23.

4. Whatmore AM, Dawson CE, Groussaud P, Koylass MS, King AC, Shankster SJ, Sohn AH, Probert WS, McDonald W. Marine mammal Brucella genotype associated with zoonotic infection. Emerg Infect Dis. 2008:14:517-8.

5. Stonner HG, Lackman D. A new species of Brucella isolated from the desert wood rat, Neotoma lepida Thomas. Am J Vet Research. 1975:18:947-51.

6. Gybby IW, Gibby AM. Host-parasite relationships with Brucella neotomae J Bacteriol. 1965:89:9-16.

7. Suárez-Esquivel M, Ruiz-Villalobos N, Jiménez-Rojas C, Barquero-Calvo E, Chacón-Díaz C, Víquez-Ruiz E, Rojas-Campos N, Baker KS, Oviedo-Sánchez G, Amuy E, Chaves-Olarte E, Thomson NR, Moreno E, Guzmán-Verri C. Human neurobrucellosis caused by Brucella neotomae. J Emerg Infect Dis. 2017;23: 997-1000. doi:10.3201/eid2306.162018.

8. Ariza J, Bosilkovski M, Cascio A, Colmenero JD, Corbel MJ, Falagas ME, Memish ZA, Roushan MR, Rubinstein E, Sipsas NV, Solera J, Young EJ, Pappas G. Perspectives for the treatment of brucellosis in the 21st century: the ioannina recommendations. PLoS Med. 2007:4:e317.

9. Cameron HS, Meyer ME. Metabolic studies on Brucella neotomae (Stoenner and Lackman). J Bacteriol. 1958;76:546-8.

10. Spink WW. The nature of brucellosis. Minneapolis: The University of Minnesota Press; 1956

11. Franco M, Mulder M, Gilman R, Smits H. Human brucellosis. Lancet Infect Dis. 2007;7:775-86

12. Crosby E, Llosa L, Miro Quesada M, Carrillo C, Gotuzzo E. Hematologic changes in brucellosis. J Infect Dis. 1984;150:419-24.

13. Guven T, Ugurlu K, Ergonul O, Celikbas AK, Gok SE, Comoglu S, Baykam N, Dokuzoguz B. Neurobrucellosis: clinical and diagnostic features. Clin Infect Dis. 2013;56:1407-12. doi:10.1093/cid/cit072.

14. Díaz R, Casanova A, Ariza J, Moriyón I. The rose Bengal test in human brucellosis: a neglected test for the diagnosis of a neglected disease. PLoS Negl Trop Dis. 2011;5:e950. doi:10.1371/journal.pntd.0000950.

15. Gardner AL, Carleton MD. A new species of Reithrodontomys, subgenus aporodon (cricetidae: neotominae), from the highlands of Costa Rica, with comments on Costa Rican and Panamanian Reithrodontomys. Bull Ame Mus of Nat Hist. 2009:331:157-82

16. Dabral N, Moreno-Lafont M, Sriranganathan N, Vemulapalli R. Oral immunization of mice with gamma-irradiated Brucella neotomae induces protection against intraperitoneal and intranasal challenge with virulent $B$. abortus 2308. PLoS One. 2014;9:e107180. doi:10.1371/journal.pone.0107180.

17. Moustafa D, Garg VK, Jain N, Sriranganathan N, Vemulapalli R. Immunization of mice with gamma-irradiated Brucella neotomae and its recombinant strains induces protection against virulent $B$. abortus, B. melitensis, and $B$. suis challenge. Vaccine. 2011;29:784-94.

\section{Submit your next manuscript to BioMed Central and we will help you at every step:}

- We accept pre-submission inquiries

- Our selector tool helps you to find the most relevant journal

- We provide round the clock customer support

- Convenient online submission

- Thorough peer review

- Inclusion in PubMed and all major indexing services

- Maximum visibility for your research

Submit your manuscript at www.biomedcentral.com/submit
Biomed Central 